\title{
Protease sensing using nontoxic silicon quantum dots
}

\author{
Xiaoyu Cheng \\ Benjamin F. P. McVey \\ Andrew B. Robinson \\ Guillaume Longatte \\ Peter B. O'Mara \\ Vincent T. G. Tan \\ Pall Thordarson \\ Richard D. Tilley \\ Katharina Gaus \\ John Justin Gooding
}




\title{
Protease sensing using nontoxic silicon quantum dots
}

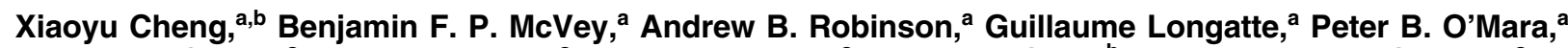 \\ Vincent T. G. Tan, ${ }^{a}$ Pall Thordarson, ${ }^{a}$ Richard D. Tilley, ${ }^{a}$ Katharina Gaus, ${ }^{b}$ and John Justin Gooding ${ }^{\mathrm{a}, *}$ \\ aUniversity of New South Wales, School of Chemistry, Australian Centre for NanoMedicine, ARC Centre of Excellence in Convergent Bio-Nano \\ Science and Technology, Sydney, New South Wales, Australia \\ bUniversity of New South Wales, EMBL Australia Node in Single Molecule Science, School of Medical Sciences, ARC Centre of Excellence in \\ Advanced Molecular Imaging, Sydney, New South Wales, Australia
}

\begin{abstract}
Herein is presented a proof-of-concept study of protease sensing that combines nontoxic silicon quantum dots (SiQDs) with Förster resonance energy transfer (FRET). The SiQDs serve as the donor and an organic dye as the acceptor. The dye is covalently attached to the SiQDs using a peptide linker. Enzymatic cleavage of the peptide leads to changes in FRET efficiency. The combination of interfacial design and optical imaging presented in this work opens opportunities for use of nontoxic SiQDs relevant to intracellular sensing and imaging. ๑ 2017 Society of Photo-Optical Instrumentation Engineers (SPIE) [DOI: 10.1117/1.JBO.22.8.087002]
\end{abstract}

Keywords: quantum dots; silicon; biosensor; Förster resonance energy transfer; self-assembled monolayer.

Paper 170270PR received Apr. 25, 2017; accepted for publication Jul. 31, 2017; published online Aug. $23,2017$.

\section{Introduction}

For a biosensor to be used in physiological conditions, ideally the information from the sensor should be obtained noninvasively and the sensor should not influence regular biological processes. Far-field optical imaging fulfills the first criterion. Colloidal quantum dots combined with far-field imaging are ideal for biosensing due to their small size, tunable surface properties, and unique optical signatures. However, the fact that many optically active colloidal nanoparticles contain toxic heavy metal elements has potentially compromised their ability to fulfill the second criterion of not perturbing the environment they are designed to monitor. ${ }^{1}$ This has led to growing interest in developing quantum dots made with materials of low toxicity, represented by group IV elements such as carbon and silicon., ${ }^{2,3}$ Currently, ultrasmall fluorescent colloidal silicon quantum dots (SiQDs) of a few nanometers in size have been shown to be benign in vivo and as a consequence they have been applied in imaging, sensing, real-time cell tracking, specific destruction of cancer cells, and drug delivery. ${ }^{4-12}$

Förster resonance energy transfer (FRET) is one of the methods by which quantum dots can be used in biosensing. ${ }^{13-17}$ This can be performed using quantum dots as either the acceptor or the donor. ${ }^{18}$ The initial example of quantum dots FRET protease sensor was reported more than a decade ago, ${ }^{13}$ but to date this concept has not been shown with nontoxic SiQDs. ${ }^{10,19}$ The difficulty with using SiQDs in biosensing has been surface modification such that silicon oxide is prevented. ${ }^{20,21}$ Preventing silicon oxide is important as silicon is an indirect bandgap semiconductor, which means the optical properties of SiQDs are sensitive to surface properties. ${ }^{22}$

The purpose of this paper is to show that SiQDs can be used as donors for FRET-based biosensors for measuring protease activity where the acceptor is an organic dye. As shown in

*Address all correspondence to: John Justin Gooding, E-mail: justin.gooding @ unsw.edu.au
Fig. 1, the SiQD FRET sensors were synthesized in a stepwise manner to demonstrate the sensing capability of SiQDs in protease sensing. The final biosensor was composed of peptide linkers attached to an SiQD donor at one end and attached to an acceptor dye DY485 at the other (referred to as an SiQDRGDC-DY485 sensor). Cleavage of a peptide by a protease enzyme resulted in removal of the dye and a diminution in FRET efficiency. Protease enzymes are the family of enzymes that perform proteolysis. They are overexpressed in most cancers, and proteolytic activity has been one of the most important sensing targets for cancer diagnosis. ${ }^{23}$ Overexpression of protease enzymes is also associated with infection, cholesterol regulations, apoptosis, and necrosis. ${ }^{23}$ In this study, trypsin was used as a model protease. Trypsin is a serine protease produced mainly in the pancreas as a proenzyme trypsinogen. ${ }^{24}$

\section{Methods and Materials}

\subsection{Chemicals and Materials}

Silicon tetrabromide $\left(\mathrm{SiBr}_{4}\right)$, tetraoctylammoniabromide (TOAB), lithium aluminum hydride $\left(\mathrm{LiAlH}_{4}, 1.0 \mathrm{M}\right.$ THF solution), 1,7-octadiene, Hoescht 33258, and 10× trypsin EDTA (1 mM) stock were purchased from Sigma-Aldrich and were used as received unless otherwise stated. RGDC peptide was purchased from Genscript. The fluorescent dye DY485-NHS was obtained from Dyomics. Dry solvents were obtained by passing through a PURE-SOLV (Innovative Technology) solvent purification system with water content below $15 \mathrm{ppm}$.

\subsection{Synthesis and Purification of 1,7-Octadiene- Passivated Silicon Quantum Dots}

Hydrogen-terminated SiQDs were prepared in accordance with a published method. ${ }^{8,25-27}$ In brief, all experiments were performed under Ar using a standard Schlenk line setup. In

1083-3668/2017/\$25.00 @ 2017 SPIE 


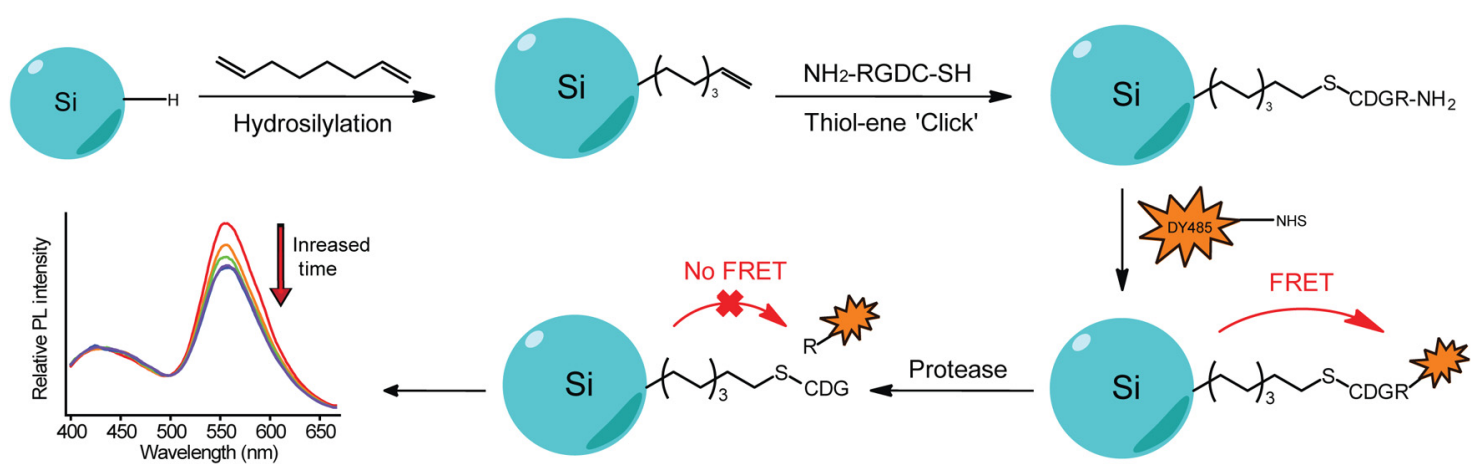

Fig. 1 Schematic of the fabrication of the FRET protease sensor based on SiQDs. As synthesized SiQDs were modified with 1,7-octadiene using UV light followed by attachment of a peptide sequence RGDC where the free thiol on the cysteine couples to the distal alkene on the monolayer modified SiQD. The dye molecule DY485-NHS was then attached to the free amine at the N-terminus of the peptide sequence. Protease activity is then determined by the change in PL as the peptide is cleaved by the enzyme.

a typical experiment, $100 \mu \mathrm{L}$ of $\operatorname{SiBr}_{4}\left(0.8 \times 10^{-3}\right.$ mole $)$ and $1.5 \mathrm{~g}$ TOAB $\left(2.7 \times 10^{-3}\right.$ mole $)$ were dissolved in $100-\mathrm{mL}$ toluene in a $250-\mathrm{mL}$ three-neck flask. The mixture was sonicated for $20 \mathrm{~min}$. An excess amount of $\mathrm{LiAlH}_{4}\left(6 \times 10^{-3}\right.$ mole, excess) was then added, and the mixture was further stirred and sonicated for $45 \mathrm{~min}$. The reaction was then quenched with dry ethanol until no bubbles were seen and kept under argon.

Alkene-terminated SiQDs were prepared by reacting 1,7octadiene with hydrogen-terminated SiQDs and then purified via size exclusion chromatography. The hydrogen-terminated SiQDs obtained in the previous step were transferred to a 250-mL quartz reaction vessel (ACE Glass Inc.) via a cannula needle under Ar. For each hydrosilylation reaction, $4 \mathrm{~mL}$ of octadiene was added, and the mixture was treated with UV $(254 \mathrm{~nm})$ for $15 \mathrm{~h}$. After the reaction, all solvent and unreacted alkene were evaporated under reduced pressure and elevated temperature, with the crude product obtained as pale-yellow oil. Then water $(3 \times 20 \mathrm{~mL})$ and hexane $(3 \times 20 \mathrm{~mL})$ were added to the mixture. The hexane layer was extracted and was passed through a PVDF membrane with $0.45-\mu \mathrm{m}$ pore size. To further purify octadiene-passivated SiQDs, size-exclusion chromatography was used. All of the hexane was evaporated and particles were resuspended in $3 \mathrm{~mL}$ of toluene. Bio-Beads SX bead was used as the stationary phase and toluene was used as the eluent with no pressure applied. Only fractions that showed blue photoluminescence (PL) under a UV lamp were collected. The column was washed with twice the amount of the beads' volume after purification.

\subsection{Preparation of SiQDs-DY485 FRET Conjugates}

For a typical thiol-ene "click" reaction on the alkene-functionalized SiQDs, the solvent containing $5 \mathrm{mg}$ of SiQDs-octadiene dispersion was first evaporated, and then $1 \mathrm{~mL}$ of peptide (sequence: N'-RGDC-C') DMSO or DMF solution at concentration of $5 \mathrm{mg} / \mathrm{mL}$ was added. The mixture was treated under UV $(4 \times 8 \mathrm{~W})$ for $5 \mathrm{~h}$ to allow sufficient coupling of the thiolene click reaction to complete. The mixture was then dialyzed with Tube-O-Dialyzer (1.5k MWCO, G-Biosciences) in $1 \times$ phosphate buffer saline (PBS) at $4^{\circ} \mathrm{C}$ for $24 \mathrm{~h}$ with the buffer replaced every $8 \mathrm{~h}$. The sample was then recovered, and DY485-NHS was added in the desired concentration for $3 \mathrm{~h}$ to allow the coupling reaction to complete.

\subsection{Characterization of Morphology and Optical Properties of SiQDs}

The size and morphology of the synthesized SiQDs were characterized by transmission electron microscopy (TEM) and dynamic light scattering (DLS) measurements. TEM measurements were performed on a Philip CM 200 microscope operated at $200 \mathrm{kV}$. All TEM images were visualized without staining. DLS measurements were performed on a Brookhaven 90 Plus Instrument. Measurements were performed at $25^{\circ} \mathrm{C}$ and intensity mean was plotted versus the size distribution. Experimental parameters used are as follows: scan time: 50; buffer condition: hexane for hydrophobic surface nanoparticles and PBS for hydrophilic surface nanoparticles; and scattering angle: $173 \mathrm{deg}$. Surface functionalization of SiQDs was characterized by FT-IR spectroscopy. FT-IR measurements were performed on an Avatar 320 FT-IR spectrometer. Measurements were done by dropping $10 \mu \mathrm{L}$ of concentrated SiQD dispersion onto a pellet premade by grinding a powder of $\mathrm{KBr}$. H-NMR measurements were performed on a Bruker $300 \mathrm{MHz}$ instrument to confirm the purity of synthesized nanoparticles. The optical properties of SiQDs were characterized by UV-vis and fluorescence spectroscopy. UV-vis spectra were recorded on a Cary-50 $\mathrm{UV}$-vis spectrometer and fluorescence spectra measured on a Cary-Eclipse fluorescence spectrometer.

\subsubsection{MTT toxicity assay}

An MTT (3-[4,5-dimethylthiazol-2-yl]-2,5 diphenyl tetrazolium bromide) assay (Sigma-Aldrich) was performed to evaluate the cellular toxicity of SiQDs. HeLa cells were cultured to $70 \%$ to $80 \%$ confluence in a 96-well plate prior to the experiment. Twenty $\mu \mathrm{L}$ of SiQDs were added to each well and incubated for $24 \mathrm{~h}$. After the incubation, the MTT reagent $(5 \mathrm{mg} / \mathrm{mL})$ was reconstituted in $1 \times \mathrm{PBS}$ and was added in an equal volume. The cells were further incubated for 2 to $3 \mathrm{~h}$. After the incubation period, the resulting formazan crystals were dissolved by adding an equal volume of DMSO to the original culture medium volume. Upon fully dissolving the crystals, the absorption measurements were performed using a FLUOstar fluorescence plate reader (BMG Labtech) at $570 \mathrm{~nm}$ and background at $690 \mathrm{~nm}$. 


\section{Results and Discussions}

\subsection{Characterization of Surface-Modified Silicon Quantum Dots}

The SiQDs were characterized extensively prior to utilizing the SiQD-RGDC-DY485 biosensors. TEM results indicated that the obtained nanoparticles were spherical in shape and relatively monodispersive with an average size of the silicon cores of $3.4 \pm 0.7$ nm [Fig. 2(a)]. High-resolution TEM images revealed lattice fringes matching the (220) lattice spacing of silicon. DLS measurements showed that the hydrodynamic size of the SiQDs was $5.4 \pm 0.6 \mathrm{~nm}$ [Fig. 2(b)]. After conjugation of the peptide, there was a slight increase in SiQDs size, with the small peak measured as $7.2 \pm 1.2 \mathrm{~nm}$ [Fig. 2(c)]. Greater amounts of aggregation were also observed after the modification process. For successful fabrication of the SiQDs FRET donor, we highlight the importance of nanoparticle purification. After each surface modification step, nanoparticles showed expected signatures with FT-IR measurements [Fig. 2(e)]. In particular, bands at 1600 at $3055 \mathrm{~cm}^{-1}$ were due to the distal alkene on the surface, whereas peaks at 3400 and $1700 \mathrm{~cm}^{-1}$ were evidence for the successful grafting of the peptides. With the protocol used, typically $\sim 10 \mathrm{mg}$ of purified SiQDs were obtained, while adding more precursors led to aggregated by products and particles were no longer monodisperse.

The progress of the surface modification could also be monitored by the solvent phases in which the SiQDs could be dispersed and from their luminescence [Fig. 2(d)]. When modified with 1,7-octadiene, the nanoparticles were only dispersible in nonpolar solvents such as hexane and naturally only the luminescence of the SiQDs was observed. After attachment of the hydrophilic peptide onto the surface, the SiQDs became dispersible in water. Further modification with dye molecules resulted in the SiQD-RGDC-DY485 sensor remaining in aqueous solution but now the luminescence changed from blue to orange upon UV excitation of $365 \mathrm{~nm}$. This color change reflected FRET from the SiQD donor to the DY485 acceptor with its characteristic emission. The optical characteristics of the SiQDRGDC-DY485 sensors were described in a conference proceedings, as is shown in Fig. 2(f). ${ }^{28}$

The organic dye acceptor used in this study, DY485, was chosen primarily because it has a large Stokes shift of $\sim 85 \mathrm{~nm}$ [Fig. 2(f)], which provides minimal overlap between the emissions of the SiQD donor and the acceptor. Hence, emission from a donor channel can be observed without interference from the acceptor. Furthermore, the absorption of DY485 exhibits good overlap with the emission peak of SiQDs as needed for a good FRET donor/acceptor pair. ${ }^{13}$ With the donor-acceptor pair presented herein, the Förster radius was calculated to be $\sim 3.2 \mathrm{~nm}$, where the spacing between the dye and the nanoparticle was estimated to be $\sim 2.5 \mathrm{~nm}$. This calculation was determined from the surface of the SiQDs as emission from SiQDs has been previously shown to most likely be interface dominated. ${ }^{22,27-29}$

\subsection{Sensor Performance}

Coupling the dye to the SiQDs resulted in FRET occurring as shown visually in Fig. 2(d). The spectral changes of the SiQDRGDC-DY485 as a function of time as the acceptor dye is attached are shown in Fig. 3(a). What is apparent from this figure is that the growth on the acceptor emission as a function of time as expected for with more dye being attached with longer (a)

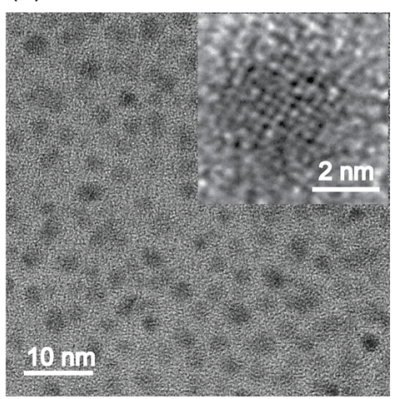

(d)

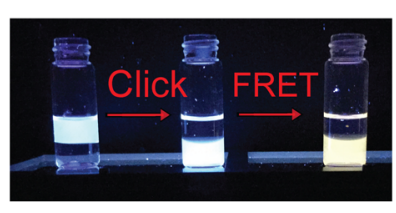

(b)

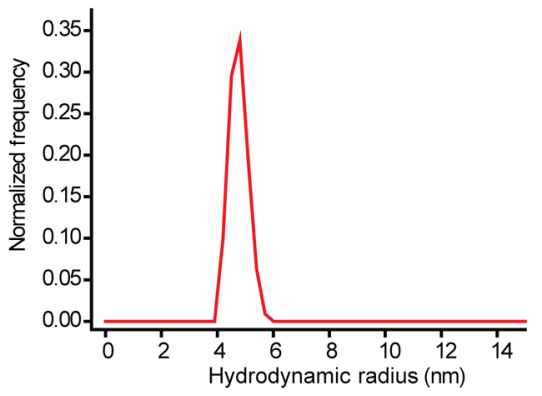

(e)

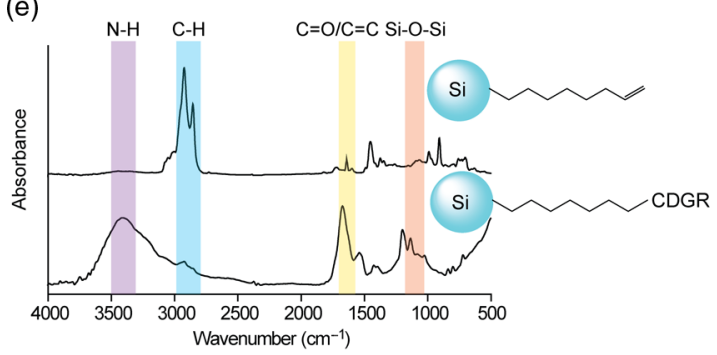

(c)
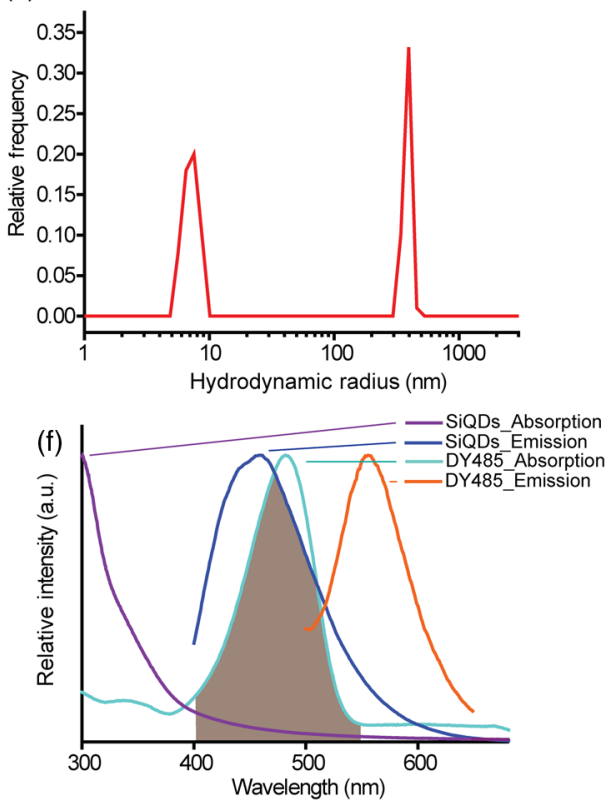

Fig. 2 Characterization of size and surfaces of SiQDs used as the FRET donors. (a) Representative TEM images and high-resolution (HR-TEM) images showing the spherical morphology and lattice fringes of nanoparticles synthesized (b, c) DLS measurements confirming the narrow size distribution of (b) alkene and (c) peptide conjugated SiQDs. (d) Picture showing the fluorescence of the nanoparticles in different phases obtained from each surface conjugation step. The nanoparticles were dispersed in solvents containing hexane (top layer) or Milli-Q water (bottom layer) under direct excitation using a 365nm UV lamp and (e) FT-IR of surface modified SiQDs. (f) Spectral overlay between SiQDs and DY485 as also indicated in Ref. 28. 

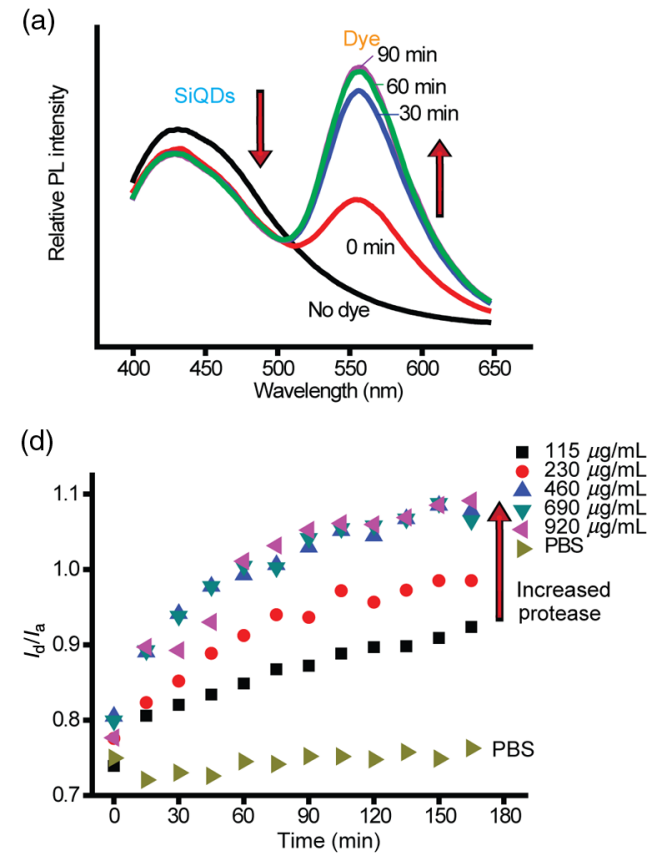

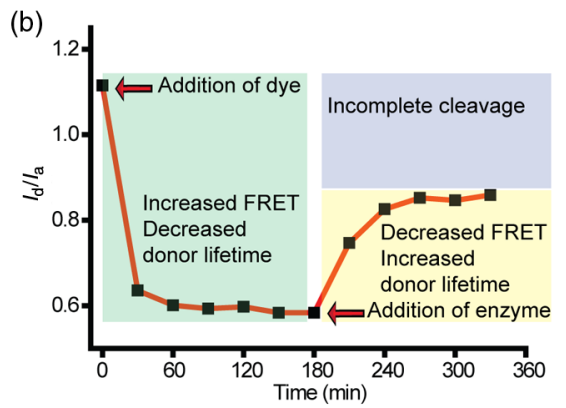

(e)

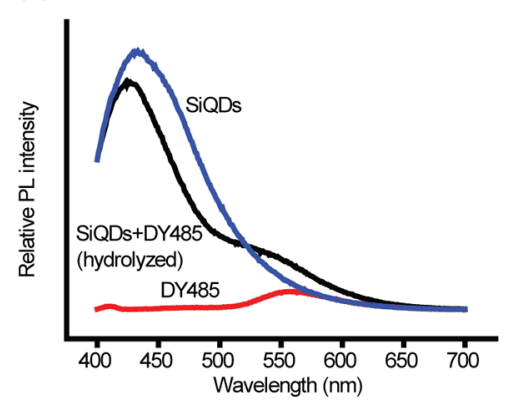

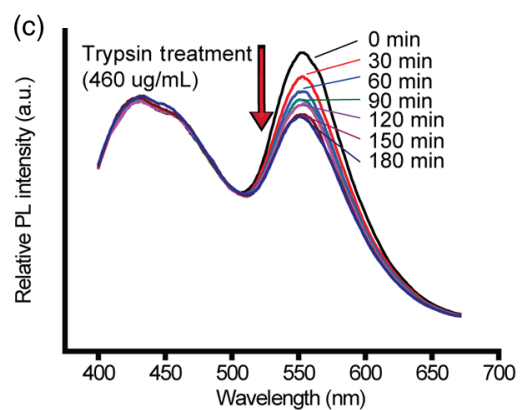

(f)

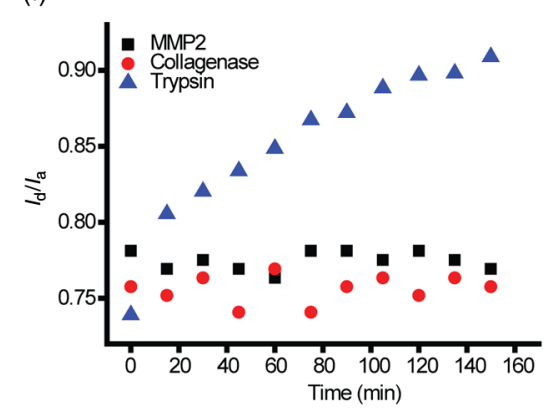

Fig. 3 Sensor performance. (a) Change of PL with time upon addition of DY485 (20 $\mu \mathrm{M})$. An immediate drop of PL was seen after the addition of the dye acceptor, while the donor peak remained largely stable throughout the rest of the reaction. Excitation wavelength: $350 \mathrm{~nm}$. (b) Typical $I_{\mathrm{d}} / I_{\mathrm{a}}$ trend monitored over the entire experiment. (c) Change of PL upon the addition of trypsin at $460 \mu \mathrm{g} / \mathrm{mL}$. (d) Enzymatic response of the SiQDs protease sensor at different trypsin concentrations. (e) Reaction with hydrolyzed DY485 showed a drop in the donor channel but no increase of intensity in the acceptor channel. (f) Enzyme specificity study using collagenase $(100 \mu \mathrm{g} / \mathrm{mL})$ and matrix metalloproteinases 2 (MMP2, $140 \mu \mathrm{g} / \mathrm{mL}$ ) which showed no effect on the FRET signal in contrast to clear change for trypsin $(115 \mu \mathrm{g} / \mathrm{mL})$. Experiments were performed with $20 \mu \mathrm{M}$ of DY485 with excitation wavelength of $350 \mathrm{~nm}$.

coupling times. Less expected was the only minor decrease in the donor peak during the coupling reaction. A closer inspection for the PL trend at the donor channel [Fig. 3(b)] showed that PL intensity ratio dropped immediately after the addition of the dye, where after the intensity remained fairly stable with less than $10 \%$ variation over the remaining reaction time. We attribute this observation to two factors. The first being that silicon is an indirect bandgap semiconductor as in contrast to conventional quantum dots which are typically made from direct bandgap materials. This has led to lower quantum yield and also a strong impact of surface properties over photophysics of the particles, as seen by us and other groups previously. ${ }^{12,22,30,31}$ The second factor is that since we did not purify the conjugate after dye coupling, the drop of signal at the donor channel could be predominated by strong absorption of the dye. Note this cannot be attributed to nonspecific adsorption of the dye because a control experiment with hydrolyzed DY485 did not increase the acceptor channel signal [Fig. 3(e)]. The response of the sensor to the addition of trypsin is shown in Figs. 3(b) and 3(c). Upon addition of the trypsin, the FRET efficiency to the accept dye decreased as indicated by the decrease in intensity of the emission peak at $550 \mathrm{~nm}$. This result suggests that trypsin has cleaved the peptide linkage between the SiQDs and the dye and hence less dye is within appreciable FRET distance. Note again the donor emission peak is insensitive to the extent of FRET. The timescale over which this cleavage occurs is shown in Figs. 3(b) and 3(f). The fact that the sensor is specific toward trypsin is seen in Fig. 3(f) where additional collagenase $(100 \mu \mathrm{g} / \mathrm{mL})$ or MMP2 $(140 \mu \mathrm{g} / \mathrm{mL})$ under the same experimental conditions resulted in no change in luminescence and hence no cleavage of the peptide linker.

\subsection{Kinetic Analysis of Enzyme Activity}

For protease sensing, the FRET conjugate was treated with trypsin, which cleaves at the $\mathrm{C}$ terminus of glycine of the peptide sequence. With an increasing amount of enzyme added, the respective PL measurements indicated a more significant change of FRET response until a maximum change was reached at $\sim 460 \mu \mathrm{g} / \mathrm{mL}$ [Fig. 3(d)]. This was in comparison to the control group treated with PBS, which did not show any appreciable change of PL throughout the experiment; thus confirming the change in FRET response was due to the cleavage of the peptide. The reaction kinetics of the enzymatic cleavage as shown in Fig. 3(d) was then investigated (Fig. 4). With the stepwise surface modification strategy used, the substrate concentration and nanoparticle/ligand ratios were not known. ${ }^{32,33}$ For this reason, a modified approach for studying the surface reaction kinetics of our system was needed. To do this we used $I_{\mathrm{d}} / I_{\mathrm{a}}$ as a function of enzyme reaction time, which was then fitted with a global nonlinear regression model using an integrated form of the Michaelis-Menten equation using Lambert function and known enzyme concentration. ${ }^{34}$

More specifically, protease reactions progress as

$E+S \rightleftarrows E S \rightarrow E+$ product.

With the rate equation given as

$v=-\frac{d}{d t}[S](t)=\frac{k_{\mathrm{cat}}\left[E_{0}\right][S](t)}{K_{m}+[S](t)}$.

Since in our case, the substrate concentration is unknown while the enzyme concentration is known, which is opposite 

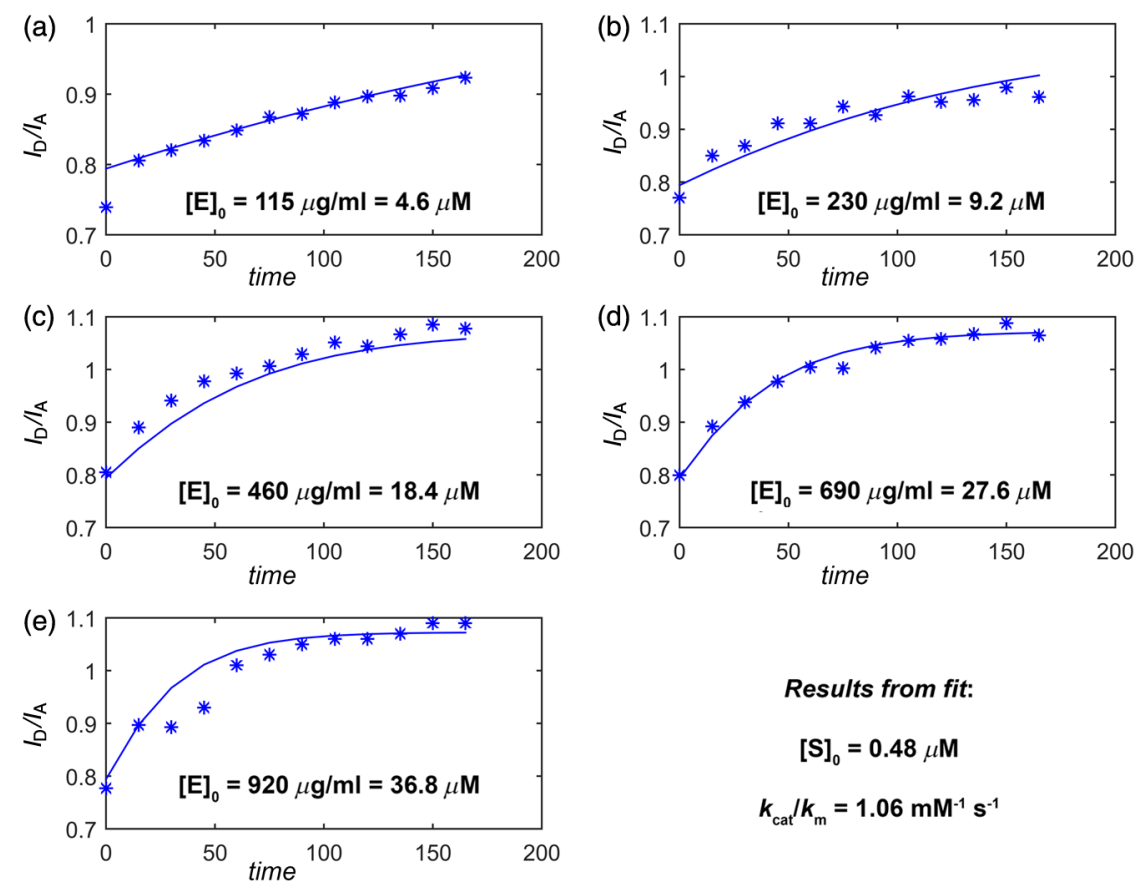

$$
\begin{gathered}
\text { Results from fit: } \\
{[\mathrm{S}]_{0}=0.48 \mu \mathrm{M}} \\
k_{\mathrm{cat}} / k_{\mathrm{m}}=1.06 \mathrm{mM}^{-1} \mathrm{~s}^{-1}
\end{gathered}
$$

Fig. 4 Enzyme kinetics. The enzymatic response of the SiQDs protease sensor showing the raw data (blue stars) versus fitted rate curves based on a global nonlinear fit to the integrated Michaelis-Menten equations with (a-e) corresponding to the different trypsin protease concentrations shown. The results from the fitting process are also shown in the lower right corner.

to the situation for conventional studies, the analysis of our data is based on direct integration of the Michaelis-Menten equation using the Lambert $W$ function as shown by Schnell and Mendoza ${ }^{34}$

$$
[\mathrm{S}]_{\mathrm{t}}=K_{m} W_{0}\left[\frac{[\mathrm{S}]_{0}}{K_{m}} e^{\left(\frac{[\mathrm{S}]_{0-V_{m} t}}{K_{m}}\right)}\right] .
$$

Here, $W_{0}$ stands for the Lambert $\mathrm{W}$ equation which can be solved by accurate straightforward numerical approaches via MATLAB ${ }^{\circledR}$. The maximum enzymatic turnover frequency, $k_{\text {cat }}$, is simply the maximum rate achieved by the system, $V_{\mathrm{m}}$, divided by $[\mathrm{E}]_{0}$, such that

$k_{\mathrm{cat}}=\frac{V_{m}}{[\mathrm{E}]_{0}} \rightarrow V_{\mathrm{m}}=k_{\mathrm{cat}}[\mathrm{E}]_{0}$.

Here, the total enzyme concentration $[\mathrm{E}]_{0}$ is known, but not the total substrate concentration $[S]_{0}$. Therefore, by inserting Eq. (4) into Eq. (3), we obtain

$[\mathrm{S}]_{\mathrm{t}}=K_{m} W_{0}\left[\frac{[\mathrm{S}]_{0}}{K_{m}} e^{\left(\frac{[\mathrm{S}]_{0-k_{\mathrm{cat}}[\mathrm{E}]_{0} t}}{K_{m}}\right)}\right]$.

An important assumption is made here, such that within the measurements range $I_{\mathrm{A}}$ is a linear function of the substrate density on the surface of SiQDs. This comes from the fact that FRET induced fluorescence changes at the donor channel due to FRET are very small. Indeed, the application of the quasisteady-state approximation to the donor-excited channel gives its concentration expression called $\left[D^{*}\right]$ as a function of the total donor concentration $[D]$ and the different rate constants $\left[k_{\text {exc }}\right.$ for the excitation, $k_{F}$ for the relaxation by fluorescence emission, $k_{\text {FRET }}$ for the relaxation by FRET to the acceptor, $k_{i}$ all other relaxation pathway (thermal relaxation and all other quenching processes), and $[\mathrm{S}]$ the substrate concentration (product of nanoparticle concentration and number of substrates per nanoparticle)]

$\left[D^{*}\right]=[D] \frac{k_{\mathrm{exc}}}{k_{\mathrm{exc}}+k_{F}+[S] k_{\mathrm{FRET}}+\sum_{i=1}^{N} k_{i}}$.

As the fluorescence is proportional to the rate of relaxation by fluorescence emission (first-order reaction with $\left[D^{*}\right]$ as reactant and $k_{F}$ as rate constant), the fact that little change in the donor channel is observed as a function of the acceptor concentration suggests that $[A] k_{\mathrm{FRET}} \ll k_{\mathrm{exc}}+k_{F}+\sum_{i=1}^{N} k_{i}$. In other words, the donor quenching observed is dominated by factors other than FRET. This lets us rewrite the last expression of the excited donor concentration which is independent of the substrate concentration

$\left[D^{*}\right]=[D] \frac{k_{\mathrm{exc}}}{k_{\mathrm{exc}}+k_{F}+\sum_{i=1}^{N} k_{i}}$.

Similarly, the excited acceptors' concentration can be expressed as

$\left[S^{*}\right]=[S] \frac{k_{\mathrm{FRET}}\left[D^{*}\right]}{k_{\mathrm{FRET}}\left[D^{*}\right]+k_{F, S}+\sum_{i=1}^{N} k_{i, S}}$.

If we assume that the intensity in the acceptor channel is proportional to $\left[S^{*}\right]$, the independence of $\left[D^{*}\right]$ with $[\mathrm{S}]$ shows that $I_{\mathrm{a}}$ is proportional to $[S]$. However, a final background value has to be added into the modeling. Using this assumption, we can now relate Eq. (8) directly to the observed $\left(I_{\mathrm{d}} / I_{\mathrm{a}}\right)$ ratio at any given time $\left(I_{\mathrm{d}} / I_{\mathrm{a}}\right)_{\mathrm{t}}$, assuming a background final value of $\left(I_{\mathrm{d}} / I_{\mathrm{a}}\right)_{\infty}$ 


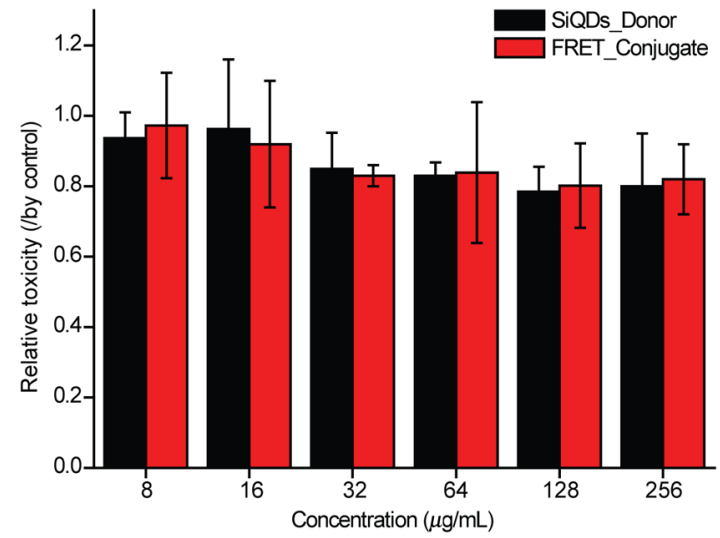

Fig. 5 Nanoparticle toxicity. MTT toxicity test after $24 \mathrm{~h}$ incubation with SiQDs in reference to control group treated with PBS. The results suggest no acute toxicity for nanoparticle concentration up to $256 \mu \mathrm{g} / \mathrm{mL}$.

and by defining the molar concentration response of $\left(I_{\mathrm{d}} / I_{\mathrm{a}}\right)$ as $\varepsilon\left[\left(I_{\mathrm{d}} / I_{\mathrm{a}}\right)\right]$, we obtain

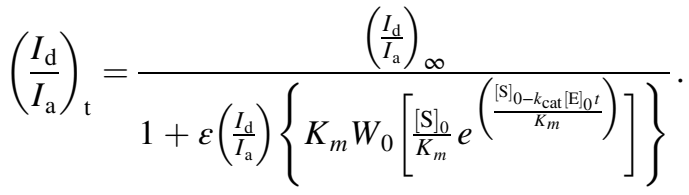

This equation was then used as the basis of a global fit for all five $\left(I_{\mathrm{d}} / I_{\mathrm{a}}\right)$ curves below.

By this method, an estimate of both $k_{\text {cat }} / K_{m}=$ $1.06 \mathrm{mM}^{-1} \mathrm{~s}^{-1}$, and the total substrate concentration $[S]_{0}=$ $0.48 \mu \mathrm{M}$ was obtained. It should be noted that with the method devised it was not possible to obtain independent values of $K_{m}$ and $k_{\text {cat }}$, but only their ratio as the results suggested [E] $\gg[E S]$. The fitting also suggested the value of $k_{\text {cat }} / K_{m}$ was about three to five times lower than the reported value for trypsin-catalyzed proteolytic cleavage of a longer peptide conjugate on conventional quantum dots. ${ }^{33}$

\subsection{Nanoparticle Toxicity}

To test nanoparticle toxicity, we performed MTT toxicity experiments with the SiQDs donor and the FRET conjugate in vitro using HeLa cells (Fig. 5). The particles were incubated with the cells overnight at different concentrations, and no apparent toxic effect was seen for particle amounts up to $256 \mu \mathrm{g} / \mathrm{mL}$ compared with the control group, where only PBS was applied. The results were consistent with previous reports showing no toxic effect of SiQDs. ${ }^{26,27,35,36}$

\section{Conclusions}

In summary, we report the first proof-of-concept study of FRET protease sensing with quantum dots made from a nontoxic material, crystalline silicon. This was achieved by a combination of materials synthesis, interfacial design, and advanced far-field imaging techniques. Mechanistic study revealed that the surface reaction follows the Michaelis-Menten kinetics model. The general applicability of wet chemistry and thiol-based surface modification strategy presented allows a simple way of preparing colloidal SiQDs sensors for immobilizing target molecules onto the surface, and the use of a microscopic method suggested measurements can be performed in cellular contexts. The concepts brought by this paper aim to bring new opportunities of bioapplications using nontoxic quantum dots in sensing and in general.

\section{Disclosures}

The authors declare no competing financial interests.

\section{Acknowledgments}

We acknowledge the generous financial support from the Australian Research Council Centre of Excellence in Convergent Bio-Nano Science and Technology (CE140100036; J.J.G. and P.T.), ARC Australian Laureate Fellowship (FL150100060; J.J.G.), ARC Centre of Excellence in Advanced Molecular Imaging (CE140100011; K.G.), and Senior Research Fellowship from the National Health and Medical Research Council of Australia (APP1059278; K.G.).

\section{References}

1. M. Bottrill and M. Green, "Some aspects of quantum dot toxicity," Chem. Commun. 47(25), 7039-7050 (2011).

2. M. Dasog et al., "Silicon nanocrystals and silicon-polymer hybrids: synthesis, surface engineering, and applications," Angew. Chem. Int. Ed. 55(7), 2322-2339 (2016).

3. X. Cheng et al., "Colloidal silicon quantum dots: from preparation to the modification of self-assembled monolayers (SAMs) for bio-applications," Chem. Soc. Rev. 43(8), 2680-2700 (2014).

4. Y. Su et al., "In vivo distribution, pharmacokinetics, and toxicity of aqueous synthesized cadmium-containing quantum dots," Biomaterials 32(25), 5855-5862 (2011)

5. J. A. Kelly et al., "Size-dependent reactivity in hydrosilylation of silicon nanocrystals," J. Am. Chem. Soc. 133(24), 9564-9571 (2011).

6. C. M. Hessel et al., "Synthesis of ligand-stabilized silicon nanocrystals with size-dependent photoluminescence spanning visible to near-infrared wavelengths," Chem. Mater. 24(2), 393-401 (2012).

7. F. Erogbogbo et al., "Biocompatible luminescent silicon quantum dots for imaging of cancer cells," ACS Nano 2(5), 873-878 (2008).

8. J. H. Warner et al., "Water-soluble photoluminescent silicon quantum dots," Angew. Chem. Int. Ed. 44(29), 4550-4554 (2005).

9. R. K. Baldwin et al., "Room temperature solution synthesis of alkyl-capped tetrahedral shaped silicon nanocrystals," J. Am. Chem. Soc. 124(7), 1150-1151 (2002).

10. Y. Su, X. Ji, and Y. He, "Water-dispersible fluorescent silicon nanoparticles and their optical applications," Adv. Mater. 28(47), 10567-10574 (2016).

11. C. Song et al., "Peptide-conjugated fluorescent silicon nanoparticles enabling simultaneous tracking and specific destruction of cancer cells," Anal. Chem. 87(13), 6718-6723 (2015).

12. X. Cheng et al., "One-pot synthesis of colloidal silicon quantum dots and surface functionalization via thiol-ene click chemistry," Chem. Commun. 48(97), 11874-11876 (2012).

13. I. L. Medintz et al., "Self-assembled nanoscale biosensors based on quantum dot FRET donors," Nat. Mater. 2(9), 630-638 (2003).

14. V. Palomo et al., "3,4-dihydroxyphenylalanine peptides as nonperturbative quantum dot sensors of aminopeptidase," ACS Nano 10(6), 6090-6099 (2016)

15. F. Erogbogbo et al., "Energy transfer from a dye donor to enhance the luminescence of silicon quantum dots," Nanoscale 4(16), 5163-5168 (2012).

16. M. Locritani et al., "Silicon nanocrystals functionalized with pyrene units: efficient light-harvesting antennae with bright near-infrared emission," J. Phys. Chem. Lett. 5(19), 3325-3329 (2014).

17. A. M. Dennis et al., "Quantum dot-fluorescent protein FRET probes for sensing intracellular pH," ACS Nano 6(4), 2917-2924 (2012).

18. W. R. Algar et al., "Quantum dots as simultaneous acceptors and donors in time-gated Förster resonance energy transfer relays: characterization and biosensing," J. Am. Chem. Soc. 134(3), 1876-1891 (2012). 
19. C. M. Gonzalez and J. G. C. Veinot, "Silicon nanocrystals for the development of sensing platforms," J. Mater. Chem. C 4(22), 4836-4846 (2016).

20. S. Ciampi, J. B. Harper, and J. J. Gooding, "Wet chemical routes to the assembly of organic monolayers on silicon surfaces via the formation of Si-C bonds: surface preparation, passivation and functionalization," Chem. Soc. Rev. 39(6), 2158-2183 (2010).

21. F. Hua, M. T. Swihart, and E. Ruckenstein, "Efficient surface grafting of luminescent silicon quantum dots by photoinitiated hydrosilylation," Langmuir 21(13), 6054-6062 (2005).

22. M. Dasog et al., "Chemical insight into the origin of red and blue photoluminescence arising from freestanding silicon nanocrystals," ACS Nano 7(3), 2676-2685 (2013).

23. C. Cordovilla and T. M. Swager, "Strain release in organic photonic nanoparticles for protease sensing," J. Am. Chem. Soc. 134(16), 6932-6935 (2012).

24. A. Schuchert-Shi and P. C. Hauser, "Peptic and tryptic digestion of peptides and proteins monitored by capillary electrophoresis with contactless conductivity detection," Anal. Biochem. 387(2), 202-207 (2009).

25. R. D. Tilley et al., "Micro-emulsion synthesis of monodisperse surface stabilized silicon nanocrystals," Chem. Commun. 0(14), 1833-1835 (2005).

26. A. Shiohara et al., "Chemical reactions on surface molecules attached to silicon quantum dots," J. Am. Chem. Soc. 132(1), 248-253 (2010).

27. X. Cheng et al., "Versatile 'click chemistry' approach to functionalizing silicon quantum dots: applications toward fluorescent cellular imaging," Langmuir 30(18), 5209-5216 (2014).

28. X. Cheng et al., "Colloidal silicon quantum dots: from preparation to the modification of self-assembled monolayers for bioimaging and sensing applications," Proc. SPIE 10078, 1007800 (2017).

29. Q. Li et al., "Silicon nanoparticles with surface nitrogen: $90 \%$ quantum yield with narrow luminescence bandwidth and the ligand structure based energy law," ACS Nano 10(9), 8385-8393 (2016).

30. M. Dasog et al., "Size vs surface: tuning the photoluminescence of freestanding silicon nanocrystals across the visible spectrum via surface groups," ACS Nano 8(9), 9636-9648 (2014).

31. X. Cheng et al., "Enhancing quantum dots for bioimaging using advanced surface chemistry and advanced optical microscopy: application to silicon quantum dots (SiQDs)," Adv. Mater. 27(40), 6144-6150 (2015).

32. S. A. Diaz et al., "Probing the kinetics of quantum dot-based proteolytic sensors," Anal. Bioanal. Chem. 407(24), 7307-7318 (2015).

33. W. R. Algar et al., "Proteolytic activity at quantum dot-conjugates: kinetic analysis reveals enhanced enzyme activity and localized interfacial 'hopping,', Nano Lett. 12(7), 3793-3802 (2012).

34. S. Schnell and C. Mendoza, "Enzymological considerations for a theoretical description of the quantitative competitive polymerase chain reaction (QC-PCR)," J. Theor. Biol. 184(4), 433-440 (1997).

35. Y. Zhong et al., "Large-scale aqueous synthesis of fluorescent and biocompatible silicon nanoparticles and their use as highly photostable biological probes," J. Am. Chem. Soc. 135(22), 8350-8356 (2013).

36. Y. Zhong et al., "Microwave-assisted synthesis of biofunctional and fluorescent silicon nanoparticles using proteins as hydrophilic ligands," Angew. Chem. Int. Ed. 51(34), 8485-8489 (2012).

Xiaoyu Cheng received his BSc (Hons) degree with first class honors in chemistry in 2010 from Australian National University. In 2015, he received his $\mathrm{PhD}$ in chemistry from the University of New South Wales, supervised by Professor Justin Gooding. The focus of his $\mathrm{PhD}$ research was on the preparation and surface modification of silicon quantum dots for bioimaging and sensing applications. His research interests are in the bionano interfaces, biophotonics, and nanophotonics.
Benjamin F. P. McVey completed his PhD at the University of New South Wales in 2017 under the supervision of Professors Richard Tilley and J. Justin Gooding. His PhD focused on the synthesis and development of low toxicity nanoparticle bioimaging agents. $\mathrm{He}$ is currently interested in the synthesis and biomedical application of both metal and semiconductor nanoparticles.

Andrew B. Robinson received his BS degree in chemistry from the University of New South Wales and is currently a PhD candidate at the University of New South Wales. His research experience is predominantly in peptide chemistry and supramolecular systems specializing in hydrogels, self-assembly, and the analysis of the biocompatibility of materials for medical application.

Guillaume Longatte received his master's degree in electrochemistry from Université Pierre et Marie Curie in 2012. He earned his PhD in Christian Amatore's group in Paris. He has been a postdoctoral research follow in the Smart Materials and Surfaces Research Group since October 2015 under the guidance of Professor Gooding. His research is focused on photochemistry with molecules of biological interest such as proteins or DNA.

Peter B. O'Mara received his BSc (Hons) degree with first class honors in chemistry in 2016 from the University of New South Wales. He is now reading for a PhD with professors Gooding and Tilley on the synthesis, surface modification, and bioapplications of quantum dots.

Vincent T. G. Tan received his bachelor's degree in medicinal chemistry from the University of New South Wales, developing responsive polymeric nanoparticles for drug delivery. Currently, he is completing his $\mathrm{PhD}$ in chemistry under the supervision of Professor J. Justin Gooding, developing 3-D printable bioinks for cell cultures. He is interested in looking for new pathways of creating self-healing printable inks for biomedical applications such as spheroid growth.

Pall Thordarson earned his BSc degree in 1996, Iceland, and PhD in 2001, Sydney. He was appointed as a senior lecturer at UNSW in 2007 to 2012, promoted to associate professor in 2013, and professor in 2017. With over 100 publications, his research interests range from nanomedicine to supramolecular and systems chemistry. He has received a number of awards, including the 2012 Le Fèvre Memorial Prize from the Australian Academy of Science for outstanding basic research in chemistry by a scientist under the age of 40 .

Richard D. Tilley is the director of Electron Microscope Unit at UNSW. His research is focused on the solution synthesis of nanoparticles for applications ranging from catalysis to biomedical imaging. He received his master's degree in chemistry from Oxford University. $\mathrm{He}$ did his PhD in the Department of Chemistry, University of Cambridge, United Kingdom, after which he was a postdoctoral fellow for 2 years at the Toshiba basic R\&D Center, Japan.

Katharina Gaus is an NHMRC senior research fellow at the University of New South Wales, head of the EMBL Australia Node in Single Molecule Science, and deputy director of the ARC Centre of Excellence in Advanced Molecular Imaging. She received her $\mathrm{PhD}$ from the University of Cambridge in 1999 and has been leading an independent research group since 2005. Her group investigates signal transduction processes in T lymphocytes with advanced fluorescence microscopy approaches.

J. Justin Gooding is an ARC Australian laureate fellow at the University of New South Wales, codirector of the Australian Centre for NanoMedicine and the New South Wales Smart Sensing Network. $\mathrm{He}$ received his DPhil degree from the University of Oxford in 1994 and has been leading an independent research group since 1999. His group specializes in surface modification for a range of applications including biosensing, drug delivery, biomaterials, and electron transfer studies. 